\title{
Drosophila suzukii avoidance of microbes in oviposition choice
}

\author{
Airi Sato ${ }^{1}$, Kentaro M. Tanaka1, Joanne Y. Yew ${ }^{2}$, Aya Takahashi, ${ }^{1,3}$ \\ ${ }^{1}$ Department of Biological Sciences, Tokyo Metropolitan University, 1-1 Minamiosawa, \\ Hachioji 192-0397, Japan \\ ${ }^{2}$ Pacific Biosciences Research Center, University of Hawai 'i at Mānoa, 1993 East West Road, Honolulu, \\ HI 96822, United States \\ ${ }^{3}$ Research Center for Genomics and Bioinformatics, Tokyo Metropolitan University, 1-1 Minamiosawa, \\ Hachioji 192-0397, Japan \\ Keywords: mechanosensory stimulus, decision-making, acetic acid bacteria, Gluconobacter, Acetobacter, \\ spotted-wing Drosophila
}

\begin{abstract}
While the majority of Drosophila species lay eggs onto fermented fruits, females of D. suzukii pierce the skin and lay eggs into ripening fruits using their serrated ovipositors. The changes of oviposition site preference must have accompanied this niche exploitation. In this study, we established an oviposition assay to investigate the effects of commensal microbes deposited by conspecific and heterospecific individuals, and showed that presence of microbes on the oviposition substrate enhances egg-laying of D. melanogaster and D. biarmipes, but discourages that of $D$. suzukii. This result suggests that a drastic change has taken place in the lineage leading to D. suzukii in how females respond to chemical cues produced by microbes. We also found that hardness of the substrate affects the response to microbial growth, indicating that mechanosensory stimuli interact with chemosensory invoked decisions to select or avoid oviposition sites.
\end{abstract}

\section{Introduction}

Oviposition site selection is a critical factor in determining the survival rate of offspring in insect species. A nutritionally suitable resource may be heavily utilized by other insects and the offspring may suffer from intense competition. The females of Drosophila suzukii Matsumura (Diptera: Drosophilidae) have the ability to pierce the skin of ripening fruits and lay eggs into 
the flesh by using serrated ovipositors [1-3]. Because many other closely related Drosophila species lay eggs onto fermented fruits, this behavior allows D. suzukii to utilize a carbohydrate-rich resource before competition becomes intense [4,5].

The behavioral shift to deposit eggs into ripening fruits must have been accompanied by changes not only in the ovipositor morphology but also in the sensory systems used to evaluate the oviposition substrate. Karageorgi et al. [6] showed that when given the choice between ripe and rotten strawberry fruits, $D$. suzukii strongly preferred ripe over rotten fruit, whereas D. melanogaster showed an opposite tendency and preferred rotten fruit, consistent with other studies $[7,8]$. In the same experiment, D. biarmipes, a closely related species of $D$. suzukii, showed no preference between ripe and rotten fruit, indicating that they are at an intermediate evolutionary stage between $D$. suzukii and D. melanogaster. It has also been shown in the same study that while D. biarmipes and D. melanogaster show similarly strong preferences for soft substrates, D. suzukii lay eggs onto both hard and soft agarose gel substrates, a pattern similar to other studies [4,9]. Therefore, these studies indicate that D. suzukii have widened the range of potential substrates to include those with different degrees of hardness and does not necessarily prefer a harder fruit surface [10-12]. Thus, hardness alone does not account for the strong preference for ripe fruits as an oviposition substrate. Other sensory modifications are also likely to underlie the radical shift to an unexploited resource in D. suzukii after divergence from the $D$. biarmipes lineage.

The evolutionary changes in the D. suzukii chemosensory system and response to attractants from ripening fruits have been documented $[6,13,14]$, but the chemical properties of possible repellent substances of fermenting fruits have not been investigated in detail. As shown in a previous study, inoculation of the substrate from D. melanogaster adults significantly reduced the number of eggs laid by D. suzukii [15]. The identity of the aversive substances left by $D$. melanogaster is not known. The deposition of aggregation pheromones is one likely factor [16-18]. Additionally, microbial populations on fermenting fruits originating from the surrounding environment as well as individuals that have visited the fruit, represent another source of aggregation signals. The presence of non-pathogenic microbes guides a wide array of behavioral decisions in insects, including adult aggregation, feeding decisions, and oviposition choice [19-23]. Partnering with commensal microbes provides several benefits for insect hosts including protection from pathogenic microbes, increased access to nutritional resources, and improved offspring survival [24]. The response of D. suzukii oviposition to the microbial environment has been largely unstudied and represents an aspect of its social and ecological interactions that may have influenced the new host exploitation in this species.

Assessing the fruit condition and making the decision to select the oviposition site 
involve an integration of multiple sensory cues. It has been shown that D. suzukii has the ability to make complex decisions between healthy and fermenting fruits depending on the availability of the resource [8]. In D. melanogaster, mechanosensory (texture) and chemosensory (taste) information are integrated to direct feeding and oviposition decisions [25-27]. It is an intriguing question as to how different sensory information is processed and integrated in $D$. suzukii in comparison to D. biarmipes and D. melanogaster, both of which have different decision making criteria for choosing oviposition sites.

In this study, we investigate the effects of commensal microbes on oviposition site preferences, both independent of and in combination with the effect of the substrate hardness, in D. suzukii, D. biarmipes and D. melanogaster. In our assay, D. suzukii exhibited a strong avoidance of microbes transferred from other flies. This response was distinct from the other two species suggesting that the behavior has evolved in the lineage leading to D. suzukii after the split from $D$. biarmipes. Furthermore, we tested the combinatorial effect of the hardness and the presence or absence of microbes on the oviposition site selection. The mechanical stimuli provided by substrate hardness superseded the influence of microbial chemical signals. We show that this property was conserved among the three species despite differential preference towards hardness and microbial stimuli.

\section{Materials and Methods}

\subsection{Fly strains}

The following strains were used to compare the ovipositon site preference: D. suzukii strain Hilo collected in Hilo, Island of Hawai'i, U.S.A. in 2017, D. biarmipes strain MYS118, collected in Mysore, India in 1981, and D. melanogaster strain Canton S BL\#9515. All the strains were maintained at $25 \pm 1{ }^{\circ} \mathrm{C}$ under the $12 \mathrm{~h}$ light: $12 \mathrm{~h}$ dark light cycle. All flies were fed with standard corn meal food mixed with yeast, glucose, and agar.

\subsection{Oviposition assay to test the preference for substrates with microbial growth}

The procedure is illustrated in figure 1. Inoculation was conducted by using D. melanogaster ( 3 to 7 days after eclosion), D. biarmipes (3 to 7 days after eclosion) or D. suzukii (7 to 14 days after eclosion). One hundred to 150 flies were placed into the inoculation chamber without anesthesia and left for $8 \mathrm{~h}$. An inoculation chamber consists of a plastic cup (100 mL, Tri-Corner Beakers) and a petri dish (57 mm diameter $\times 16 \mathrm{~mm}$ height, IWAKI 1010-060) filled with $5 \mathrm{~mL}$ 1\% agar (Drosophila agar type II, Apex) in apple juice (SUNPACK, JAN code: 4571247510950 ) diluted to 
$50 \%$. No flies were placed into the control inoculation chamber. After inoculation, the surface of the substrate was washed with $1 \mathrm{~mL}$ distilled water by pipetting 10 times. Wash solutions (100 $\mu \mathrm{L}$ ) from inoculated or control plates (figure 1a) were spread onto a new agar plate $(40 \mathrm{~mm}$ diameter $\times 13 \mathrm{~mm}$ height, Azunol 1-8549-01) and incubated for $24 \mathrm{~h}$ at $25 \pm 1{ }^{\circ} \mathrm{C}$. Microbial colonies were visible on the media spread with aqueous solution from the inoculated media after $24 \mathrm{~h}$ incubation.

The oviposition assay was conducted with a petri dish chamber $(150 \mathrm{~mm}$ diameter $\times 20$ mm height, IWAKI 3030-150) containing four $\Phi 40 \mathrm{~mm}$ petri dishes with two types of media placed alternatively (figure 1b). Twenty females and 10 males were placed into the chamber without anesthesia within $3 \mathrm{~h}$ before the dark cycle and kept for $16 \mathrm{~h}$ in the dark condition. The assay was conducted under the condition of $25 \pm 1^{\circ} \mathrm{C}$ and $50 \pm 5 \%$ relative humidity. The photo image of each petri dish with substrate was taken by a camera (Olympus DP73) with transmitted light from the bottom. The number of eggs on each substrate was counted.

The preference index (PI) for the substrate with microbial growth was calculated by using the following formula:

$$
\text { Preference index (PI) for substrate with microbial growth }=\frac{N_{\text {inoculated }}-N_{\text {control }}}{N_{\text {inoculated }}+N_{\text {control }}},
$$

where $N$ inoculated and $N$ control are the total numbers of eggs on the substrates with microbial growth and the control plates, respectively.

To confirm that the PI measurements for substrates inoculated with microbial colonies reflect the activity of microbes, collected solutions from the inoculated media were filter sterilized using a syringe filter $\left(0.22 \mu \mathrm{m}\right.$ Millex ${ }^{\circledR}{ }^{-G V}$ Filter Unit). After washing the surface of the inoculated medium by repeatedly pipetting $1.2 \mathrm{~mL}$ distilled water 10 times, the aqueous solution was filtered and used in the oviposition assay as described above.

\subsection{Oviposition preference assay for substrate hardness, with and without microbes}

Inoculant from D. melanogaster was collected from three inoculation chambers, pooled, and divided into 24 ( $8 \times 3$ species) $\Phi 40 \mathrm{~mm}$ petri dishes with medium. Plates without any solution were used for the assays that did not test microbial inoculation. The remaining steps were the same as in 2.2. The PI for the soft substrate was calculated by using the following formula:

$$
\text { Preference index (PI) for soft substrate }=\frac{N_{1 \% \text { agar }}-N_{3 \% \text { agar }}}{N_{1 \% \text { agar }}+N_{3 \% \text { agar }}},
$$

where $N_{1 \%}$ agar and $N_{3 \%}$ agar are the total numbers of eggs on the $1 \%$ and $3 \%$ agar media, respectively.

\subsection{S-rRNA gene sequencing of microbial colonies used for the oviposition assays}


In order to collect the microbes tested for the oviposition assays, the surface of the inoculated substrate was washed with distilled water as described above. The solution was diluted to 200 $\mu \mathrm{L}$ total volume and spread onto a petri dish (90 mm diameter×16 mm height, IWAKI SH90-15) filled with $10 \mathrm{~mL}$ apple juice agar as described above. The media were incubated for 24 to 40 hours at $25 \pm 1{ }^{\circ} \mathrm{C}$ and single colonies were selected randomly for DNA extraction. Each colony was picked with a $10 \mu \mathrm{L}$ pipette tip, suspended in $20 \mu \mathrm{L}$ of sterile water, and incubated for 15 min at $95^{\circ} \mathrm{C}$ after adding $20 \mu \mathrm{L} 100 \mathrm{mM} \mathrm{NaOH}$. Then, $4.4 \mu \mathrm{L}$ of $1 \mathrm{M}$ Tris- $\mathrm{HCl} \mathrm{pH} 7.0$ was added to each sample and used as template DNA.

Colony PCR was performed with 16S-rRNA universal primers $8 \mathrm{~F}$ (AGAGTTTGATCMTGGCTCAG) [28,29] and 1492R (GGYTACCTTGTTACGACTT) [30,31] in a $30 \mu \mathrm{L}$ reaction using Ex Taq (TaKaRa). Amplification condition for the PCR included an initial denaturation step of $95^{\circ} \mathrm{C}$ for $3 \mathrm{~min}$, followed by 35 cycles of $95^{\circ} \mathrm{C}$ for $30 \mathrm{~s}, 53$ or $55^{\circ} \mathrm{C}$ for $30 \mathrm{~s}$, and $72{ }^{\circ} \mathrm{C}$ for $60 \mathrm{~s}$, and a final extension step of $72{ }^{\circ} \mathrm{C}$ for $5 \mathrm{~min}$. Reaction products were checked for size and purity on 1\% agarose gel and were sequenced after purification by using either BrilliantDye Terminator Cycle Sequencing Kit v2.1 (Nimagen) and a 3130 xl DNA Analyzer (Thermo Fisher Science) or BigDye Terminator v3.1 Cycle Sequencing Kit (Thermo Fisher Science) and a 3170xl DNA Analyzer (Thermo Fisher Science). Sequences were aligned by using MEGA7 [32] and trimmed from the nucleotide positions 61 to 628 of the Escherichia coli reference sequence (CP023349.1:226,883-228,438). The genus level identity of each sequence was assigned by the highest score entries in the NCBI database, "16S ribosomal RNA (Bacteria and Archaea type strains)" (as of May 28, 2020) by local BLAST (BLAST+ 2.10.0).

\section{Results}

The oviposition site preference of $D$. suzukii for ripening fruits relies on shifts in mechanosensation as well as chemosensation [6]. Recent work has shown that consistent with their preference towards ripening fruits over fermenting fruits, D. suzukii females tend to lay more eggs on non-inoculated media compared to media inoculated by D. melanogaster [15]. Our study focused on determining whether microbial presence and the hardness of the oviposition substrate form the basis of D. suzukii oviposition decisions.

\subsection{Oviposition site preference against the presence of microbes}

Oviposition can be influenced by pheromones or microbial presence. To distinguish between these two possibilities, we first established a method to test only the contribution of microbial 
growth to oviposition site preference. A water wash was used to collect substances deposited by adult flies and the inoculum was applied to sterile media (figure 1a). Many of the known pheromones used for Drosophila chemical communication are hydrophobic hydrocarbons, wax esters and alcohols [33], and are thus, not soluble in water and unlikely to be transferred in the water wash. After incubation, microbial colonies were visible on the inoculated media. Media that had been exposed to water wash from control chambers did not have visible colonies.

The results from the oviposition assay on soft medium (1\% agar) indicated that $D$. suzukii avoided oviposition substrates with microbial colonies (figure $2 a$, Table S1). Given a choice between substrates with aqueous solutions from inoculated and non-inoculated media, the D. suzukii preference index (PI) was significantly less than 0 , indicating that the microbial growth discouraged oviposition. By contrast, D. melanogaster preferred ovipositing on substrates with microbial growth (figure $2 a$ ), indicating that the presence of microbes positively influenced the choice of oviposition site for this species. To trace the evolutionary trajectory of this preference, we also conducted the same experiments using D. biarmipes, a closely related species to D. suzukii. Remarkably, as with D. melanogaster, the microbes positively influenced oviposition site choice of $D$. biarmipes (figure $2 a$ ) indicating that the preference for ovipositing at sites with commensal microbes is the ancestral state among these species and that $D$. biarmipes still retain this characteristic. These results were consistent when using microbes from conspecific and heterospecific inoculation (figure $2 a$ ). Thus, the drastic change from attraction to avoidance of microbes is predicted to have occurred in the lineage leading to D. suzukii after the separation from the D. biarmipes lineage.

To confirm that the presence of microbes in the water wash is the primary factor in guiding oviposition, we passed the collected aqueous solution through a $0.22 \mu \mathrm{m}$ filter to remove microbes and large food particles while keeping nutrients, metabolites, and other small molecules found in feces. In all species, filter-sterilization of the inoculant eliminated both positive and negative oviposition preferences (figure $2 b$, Table S2). Therefore, microbes that can be removed by a $0.22 \mu \mathrm{m}$ filter are likely to be the main factor affecting oviposition site preferences.

To identify the main bacterial species that were present in the water washes of inoculated media, we sampled microbial colonies from the medium after $24 \mathrm{~h}$ of growth and performed PCR amplification of the 16S-rRNA gene sequence. The bacterial species classified at the genus level and the frequencies estimated from the sampled colonies are shown in figure S1 and Table S3-S5. The bacteria used for our oviposition preference assay were mostly from the Acetobacter and Gluconobacter genera. 
bioRxiv preprint doi: https://doi.org/10.1101/2020.09.05.284166; this version posted September 5, 2020. The copyright holder for this preprint (which was not certified by peer review) is the author/funder, who has granted bioRxiv a license to display the preprint in perpetuity. It is made available under aCC-BY-NC 4.0 International license.

\subsection{Combinatorial effect of the presence of microbes and the hardness of the oviposition substrate}

In addition to chemosensory signals, another factor that is known to affect Drosophila oviposition site preference is the hardness of the substrate. Choice assays using agarose media with different degree of hardness have shown that D. suzukii females exhibit a much weaker preference towards softer substrates compared to D. biarmipes and D. melanogaster [6]. In order to investigate the combinatorial effect of hardness and microbial growth, we conducted choice assays using hard oviposition substrate ( $3 \%$ agar medium) with and without the presence of microbes (figure 2c, Table S6).

When substrates were hard, D. melanogaster and D. biarmipes showed a PI close to 1 , which is indicative of even stronger preferences for ovipositing on media with microbial growth than when using 1\% agar media (figure 2a). Interestingly, the aversion to substrates with microbial growth exhibited by D. suzukii was reduced when the harder 3\% media were used. No significant preference or aversion was detected (figure 2c). From the outcome of this combinatorial assay, it was clear that the hardness of the substrate modifies the preferences against microbes.

Next, we investigated whether the choice between soft (1\%) and hard (3\%) agar media was affected by the presence of microbes (figure $3 a$ ). Our experimental results using $1 \%$ and $3 \%$ agar media without microbes were consistent with a previous study showing that D. suzukii has no or only a slight preference for softer media, in contrast to the strong preference exhibited by the other two species (figure 3b, Table S7). Interestingly, whether the microbes were present or not did not affect the PI between soft and hard substrates in D. melanogaster and D. suzukii. The preference towards the softer substrate became significantly weaker when microbes were present than when they were absent in D. biarmipes, but only slightly. These results indicate that rather than the presence or absence of microbial growth, the hardness of the substrate is the dominant factor in oviposition site selection.

\section{Discussion}

\subsection{Commensal microbes deposited by flies affect oviposition site preferences in D. suzukii, D. biarmipes,}

\section{and D. melanogaster, and the preference of $D$. suzukii is distinct from that of the other species}

Fruit flies like many other insects coexist with a community of gut microbes, the composition of which can vary to a large extent due to various field and laboratory conditions[34-37]. To elucidate whether gut microbes function as intra- or inter-specific behavioral cues, we examined the influence of fly-deposited microbes on oviposition behavior. 
Our results show that egg-laying decisions in Drosophila are strongly influenced by the presence of microbial growth, suggesting that microbe-derived cues influence egg-laying decisions in species that use fruit as an oviposition substrate. D. suzukii avoided media inoculated with commensal microbes, in contrast to D. melanogaster and D. biarmipes, both of which showed strong preferences toward microbe-rich media (figure 2). The reversal in preference must have occurred in the $D$. suzukii lineage after the split from $D$. biarmipes consistent with the timing of the host shift to ripening fruits. Therefore, the radical change in microbial preference may have been associated with the new niche exploitation in this lineage.

\subsection{Acid producing bacteria differentially affect oviposition behavior amongst Drosophila species}

The bacterial species used for oviposition preference assays consisted mainly of Acetobacter and Gluconobacter, both members of the acid-producing Acetobacteraceae family commonly found in the guts of lab-raised and wild fruit fly species [35] including D. suzukii $[38,39]$. Acid-producing bacteria provide benefits for host flies by accelerating growth and offering protection from pathogenic bacteria [40,41]. The colonies grown on the media are not likely to represent the actual composition of fly-associated microbiota in the wild since growth is restricted by diet and the type of media used (agar in apple juice). Flies from natural populations exhibit a more diverse microbiome [36,42]. In addition, our characterization of the microbiome focused only on bacterial species. It is likely that yeast, which are a common symbiont for drosophilids [43], are also part of the inoculum and contribute to oviposition preference [44].

D. melanogaster, biarmipes, and suzukii exhibited different proportions of Acetobacter and Gluconobacter (figure S1, Table S3-S5). However, there were no differences in the responses of the three Drosophila species to conspecific or heterospecific inoculants, indicating that both Acetobacter and Gluconobacter have similar effects on the oviposition site choice (figure 2). While D. suzukii showed a clear aversion for ovipositing on inoculated media, the response of females to Gluconobacter volatiles may be context-dependent. A previous study showed that females starved for $24 \mathrm{~h}$ exhibit clear attraction to Gluconobacter in an olfactometer bioassay [45]. Taken together with our observation that D. suzukii avoids egg-laying in the presence of Gluconobacter colonies, it is clear that reproductive and feeding site preferences can be clearly decoupled in this species. Microbial cues that are attractive for feeding may be aversive for oviposition.

\subsection{Chemical cues mediating the differential preference against microbes await further investigation}

In studies searching for oviposition deterrents for the pest management of fruit crops, at least two chemicals, geosmin and octenol (1-octen-3-ol), both of which are components of volatile 
metabolites from microorganisms present in rotting fruits, induced aversive responses in $D$. suzukii [46]. However, because these chemicals are known repellents in D. melanogaster as well $[47,48]$, the aversion to these microbial compounds is not likely to underlie the D. suzukii specific shift in oviposition site.

A study using D. melanogaster indicated that female oviposition is guided by sucrose, a gustatory cue used to sense fermentation by lactic acid-producing Enterococci bacteria [49]. Interestingly, the olfactory system was shown to be dispensable for ovipositional attraction to these microbes. In contrast, the inhibition of synaptic transmission in sweet sensing gustatory neurons, Gr5a and Gr64a neurons, impaired the oviposition preference toward fermentation sources. Whether sucrose sensing also mediates the avoidance of acetic acid bacteria in $D$. suzukii would be an intriguing question to pursue. Nevertheless, Silva-Soares et al. [4] showed that D. suzukii and D. biarmipes have similar oviposition preferences toward sites with a low protein (yeast) to carbohydrate (sucrose) ratio, suggesting that a differential response to sucrose is not likely to explain the contrasting response to acetic acid bacteria products. The microbe-derived chemical cues that govern oviposition response await further investigation.

\subsection{Oviposition site hardness supersedes the $D$. suzukiiaversion to microbial presence}

Integration of different types of stimuli is essential for critical decision-making processes such as the selection of egg deposition sites, a choice that has large influences on the early life performances of the offspring. In D. melanogaster, neural circuits governing oviposition site combine information from different modalities [50,51]. Recently, several studies [26,27] elucidated an underlying molecular mechanism for integrating mechanosensory and chemosensory information to make egg-laying decisions in D. melanogaster. Our results reveal that two different classes of sensory cues, substrate hardness and the presence of microbes, are integrated in D. suzukii oviposition decisions in a manner that is distinct from D. biarmipes and D. melanogaster (figure 2 and 3). The avoidance of microbes displayed by D. suzukii was evident only in the context of a soft substrate (figure $2 a$ ) but not a hard one (figure $2 c$ ). These results suggest that mechanical cues from surface hardness take precedence over decisions guided by microbial cues. By contrast, the preference exhibited by both D. melanogaster and D. biarmipes towards microbe-inoculated surfaces strengthened when hard substrates were used (figure $2 c$ ), indicating a similar integration of mechanical and microbial chemical cues. Conversely, microbial presence did not affect the choice between hard and soft substrates in all the three species (figure 3).

These results indicate that mechanical and chemical stimuli are not processed additively in these species. The surface hardness modifies the response to microbial cues but 
not vice versa. It remains to be determined whether surface texture is prioritized in the context of pathogenic microbes. Interestingly, previous studies showed that in female D. melanogaster, the presence of chemicals, sucrose and/or fruit juice ingredient obviate the preference for ovipositing on softer surfaces $[26,27]$. The discrepancy between the direction of interference between mechanical and chemical stimuli suggests that the hierarchy of cues used in oviposition may depend on the nature of the chemical stimulus.

\subsection{The integration of mechanical cues and microbial stimuli is conserved in oviposition choice and reflect differences in ecology}

Our findings in this study can be interpreted in the context of natural ecology of D. suzukii. In early fruiting season when all the fruits are hard or have no microbial cues, D. suzukii females may lay eggs onto any available fruits. This scenario is consistent with the results of our assays using only hard substrate (figure 2c) or only non-inoculated substrates (figure 3). During the ripening period when fruits become softer and ripe, the females may choose fruits with weaker fermentation cues in order to avoid competition with other species, which is consistent with our results using only soft substrate (figure $2 a$ ). In late fruiting season when the majority of the fruits are on the ground and rotten, the females may readily lay eggs onto suboptimal fermenting fruits, the situation resembling our assays using only inoculated substrates (figure 3). These explanations are consistent with the study by Kienzle et al. [8], which showed that $D$. suzukii exhibit stronger preferences toward ovipositing in healthy fruits when healthy and fermenting fruits are both abundant compared to when the former are less abundant. The context dependent optimization through seasonal change in host fruit condition might explain the evolutionary background of our findings where substrate hardness takes precedence over microbial presence in the decision to oviposit in this species.

Although surface hardness interacts with the response to commensal microbe cues in $D$. biarmipes and D. melanogaster as in D. suzukii, there may be some qualitative differences in ecological context between these species. D. biarmipes and D. melanogaster show a strong preference toward soft substrates inoculated with microbes, and their preferences for microbes is enhanced when the substrate is hard (figure 2). In the field, it may be the case that flies are more likely to use hard fruits in the presence of a microbial signature, which may be indicative of ongoing fermentation. In contrast to D. suzukii, both D. biarmipes and D. melanogaster tend to prefer soft substrates even when all the substrates in the vicinity have microbial growth (figure 3 ), indicating that mechanical cues supersede microbial presence in oviposition site selection. Therefore, D. suzukii may have rapidly adjusted the manner in which mechanical and chemical 
stimuli are integrated to optimize an egg-laying strategy that is different from other closely related species.

\section{Acknowledgments}

We are grateful to K. Nakayama and N. Yoneishi for excellent technical assistance, the UHM Microbial Genetics and Analytical Laboratory for use of facilities, Eurofins Genomics K.K. for Sanger sequencing, and members of the Takahashi lab and Yew lab for helpful discussions.

\section{Funding Statement}

This work was supported by JSPS KAKENHI (Grant No. JP19H03276) awarded to A.T;; Department of Defense United States Army Research Office (Grant No. W911NF1610216) and the National Institutes of Health (Grant No. 1P20GM125508) awarded to JYY.

\section{Data Accessibility}

The datasets supporting this article have been uploaded as part of the Supplementary Material.

\section{Competing Interests}

We have no competing interests.

\section{Authors' Contributions}

A.S., J.Y.Y., and A.T. conceived the research and designed the experiments. A.S. performed the experiments. A.S. and K.M.T. analysed the data. A.S. and A.T. drafted the manuscript. All authors gave final approval for publication.

\section{References}

1. Walsh DB, Bolda MP, Goodhue RE, Dreves AJ, Lee J, Bruck DJ, Walton VM, O'Neal SD, Zalom FG. 2011 Drosophila suzukii (Diptera: Drosophilidae): Invasive pest of ripening soft fruit expanding its geographic range and damagepotential. J. Integr. Pest Manag. 2, G1-G7. (doi:10.1603/PM10010)

2. Atallah J, Teixeira L, Salazar R, Zaragoza G, Kopp A. 2014 The making of a pest: the evolution of a fruit-penetrating ovipositor in Drosophila suzukiia and related species. Proc. R. Soc. London B Biol. Sci. 281. (doi:10.1098/rspb.2013.2840)

3. Muto L, Kamimura Y, Tanaka KM, Takahashi A. 2018 An innovative ovipositor for niche exploitation impacts genital coevolution between sexes in a fruit-damaging Drosophila. Proc. R. Soc. B Biol. Sci. 285, 20181635. (doi:10.1098/rspb.2018.1635)

4. Silva-Soares NF, Nogueira-Alves A, Beldade P, Mirth CK. 2017 Adaptation to new nutritional environments : larval performance , foraging decisions, and adult oviposition choices in Drosophila suzukii. BMCEcol. , 1-13. (doi:10.1186/s12898-017-0131-2)

5. Young Y, Buckiewicz N, Long TAF. 2018 Nutritional geometry and fitness consequences in Drosophila suzukii, the Spotted-Wing Drosophila. Ecol. Evol. 8, 28422851. (doi:10.1002/ece3.3849)

6. Karageorgi M, Bräcker LB, Lebreton S, Minervino C, Cavey M, Siju KP, Grunwald Kadow IC, Gompel N, Prud'homme B. 2017 Evolution of multiple sensory systems drives novel egg-laying behavior in the fruit pest Drosophila suzukii. Curr. Biol.27, 847-853. (doi:10.1016/j.cub.2017.01.055)

7. Lee JC, Bruck DJ, Curry H, Edwards D, Haviland DR, Van Steenwyk RA, Yorgey BM. 2011 The susceptibility of small fruits and cherries to the spotted-wing Drosophila, Drosophila suzukii. Pest Manag. Sci. 67, 1358-1367. (doi:10.1002/ps.2225) 
bioRxiv preprint doi: https://doi org/10.1101/2020 09.05.284166; this version posted September 5, 2020. The copyright holder for this preprint (which was not certified by peer review) is the author/funder, who has granted bioRxiv a license to display the preprint in perpetuity. It is made available under aCC-BY-NC 4.0 International license.

\section{Rep. 10, 3132. (doi:10.1038/s41598-020-59595-y)}

Guo L, Zhou Z-D, Mao F, Fan X-Y, Liu G-Y, Huang J, Qiao X-M. 2020 Identification of potential mechanosensitive ion channels involved in texture discrimination during Drosophila suzukiiegg-laying behaviour. Insect Mol. Biol. (doi:10.1111/imb.12654) Burrack HJ, Fernandez GE, Spivey T, Kraus DA. 2013 Variation in selection and utilization of host crops in the field and laboratory by Drosophila suzukiiMatsumara (Diptera: Drosophilidae), an invasive frugivore. Pest Manag. Sci. 69, 1173-1180. (doi:10.1002/ps.3489)

11. Kinjo H, Kunimi Y, Ban T, Nakai M. 2013 Oviposition Efficacy of Drosophila suzukii (Diptera: Drosophilidae) on Different cultivars of blueberry. J. Econ. Entomol. 106, 1767-1771. (doi:10.1603/ec12505)

12. Lee JC, Dalton DT, Swoboda-Bhattarai KA, Bruck DJ, Burrack HJ, Strik BC, Woltz JM, Walton VM. 2016 Characterization and manipulation of fruit susceptibility to Drosophila suzukii. J. Pest Sci. (2004). 89, 771-780. (doi:10.1007/s10340-015-0692-9)

13. Keesey IW, Knaden M, Hansson BS. 2015 Olfactory specialization in Drosophila suzukii supports an ecological shift in host preference from rotten to fresh fruit. J. Chem. Ecol. 41, 121-128. (doi:10.1007/s10886-015-0544-3)

14. Revadi S et al. 2015 Olfactory responses of Drosophila suzukiffemales to host plant volatiles. Physiol. Entomol. 40, 54-64. (doi:10.1111/phen.12088)

15. Shaw B, Brain P, Wijnen H, Fountain MT. 2018 Reducing Drosophila suzukii emergence through inter-species competition. Pest Manag. Sci. 74, 1466-1471. (doi:10.1002/ps.4836)

Lin C-C, Prokop-Prigge KA, Preti G, Potter C. 2015 Food odors trigger Drosophila males to deposit a pheromone that guides aggregation and female oviposition decisions. Elife4, 1-26. (doi:10.7554/elife.08688)

17. Xu P, Atkinson R, Jones DNM, Smith DP. 2005 Drosophila OBP LUSH is required for activity of pheromone-sensitive neurons. Neuron 45, 193-200. (doi:10.1016/j.neuron.2004.12.031)

18. Tait $\mathrm{G}$ et al. 2020 Reproductive site selection: evidence of an oviposition cue in a highly adaptive dipteran, Drosophila suzukii (Diptera: Drosophilidae). Environ. Entomol. 49, 355-363. (doi:10.1093/ee/nvaa005)

19. Lewis Z, Lizé A. 2015 Insect behaviour and the microbiome. Curr. Opin. insect Sci. 9, 86-90. (doi:10.1016/j.cois.2015.03.003)

20. Fischer CN, Trautman EP, Crawford JM, Stabb E V, Handelsman J, Broderick NA. 2017 Metabolite exchange between microbiome members produces compounds that influence Drosophilabehavior. Elife6. (doi:10.7554/elife.18855)

21. Wong AC-N, Wang Q-P, Morimoto J, Senior AM, Lihoreau M, Neely GG, Simpson SJ, Ponton F. 2017 Gut microbiota modifies olfactory-guided microbial preferences and foraging decisions in Drosophila. Curr. Biol. 27, 2397-2404.e4. (doi:10.1016/j.cub.2017.07.022) Leitão-Gonçalves R et al. 2017 Commensal bacteria and essential amino acids control food choice behavior and reproduction. PLoS Biol. 15, e2000862. (doi:10.1371/journal.pbio.2000862) Jose PA, Ben-Yosef M, Jurkevitch E, Yuval B. 2019 Symbiotic bacteria affect oviposition behavior in the olive fruit fly Bactrocera oleae. J. Insect Physiol. 117, 103917. (doi:10.1016/j.jinsphys.2019.103917)

24. Douglas AE. 2015 Multiorganismal insects: diversity and function of resident microorganisms. Annu. Rev. Entomol. 60, 17-34. (doi:10.1146/annurev-ento-010814-020822)

Jeong YT, Oh SM, Shim J, Seo JT, Kwon JY, Moon SJ. 2016 Mechanosensory neurons control sweet sensing in Drosophila. Nat. Commun. 7, 1-9. (doi:10.1038/ncomms12872)

Wu SF, Ja YL, Zhang YJ, Yang CH. 2019 Sweet neurons inhibit texture discrimination by signaling TMC-expressing mechanosensitive neurons in Drosophila. Elife 8, 1-24. (doi:10.7554/elife.46165)

27. Zhang L, Yu J, Guo X, Wei J, Liu T, Zhang W. 2020 Parallel mechanosensory pathways direct oviposition decision-making in Drosophila. Curr. Biol. , 1-14. (doi:10.1016/j.cub.2020.05.076)

Weisburg WG, Barns SM, Pelletier DA, Lane DJ. 1991 16S ribosomal DNA amplification for phylogenetic study. J. Bacteriol. 173, 697-703. (doi:10.1128/jb.173.2.697-703.1991)

Turner S, Pryer KM, Miao VP, Palmer JD. 1999 Investigating deep phylogenetic relationships among cyanobacteria and plastids by small subunit rRNA sequence analysis. J. Eukaryot. Microbiol. 46, 327-338. (doi:10.1111/j.1550-7408.1999.tb04612.x) Edwards U, Rogall T, Blöcker H, Emde M, Böttger EC. 1989 Isolation and direct complete nucleotide determination of entire genes. Characterization of a gene coding for 16 S ribosomal RNA. Nucleic Acids Res. 17, 7843-7853. (doi:10.1093/nar/17.19.7843)

31. Loy A, Lehner A, Lee N, Adamczyk J, Meier H, Ernst J, Schleifer K-H, Wagner M. 2002 Oligonucleotide microarray for 165 rRNA gene-based detection of all recognized lineages of sulfate-reducing prokaryotes in the environment. Appl. Environ. Microbiol. 68, 5064-5081. (doi:10.1128/aem.68.10.5064-5081.2002) Kumar S, Stecher G, Tamura K. 2016 MEGA7: Molecular Evolutionary Genetics Analysis version 7.0 for bigger datasets. Mol. Biol. Evol. 33, 1870-1874. 
bioRxiv preprint doi: https://doi org/10.1101/2020 09.05.284166: this version posted September 5, 2020. The copyright holder for this

preprint (which was not certified by peer review) is the author/funder, who has granted bioRxiv a license to display the preprint in perpetuity. It is made available under aCC-BY-NC 4.0 International license.

(doi:10.1093/molbev/msw054)

33. Yew JY, Chung H. 2017 Drosophila as a holistic model for insect pheromone signaling and processing. Curr. Opin. insect Sci. 24, 15-20. (doi:10.1016/j.cois.2017.09.003)

34. Bing X, Gerlach J, Loeb G, Buchon N. 2018 Nutrient-Dependent Impact of Microbes on Drosophila suzukii development. MBio 9, e02199-17. (doi:10.1128/mBio.02199-17)

35. Broderick NA, Lemaitre B. 2012 Gut-associated microbes of Drosophilamelanogaster. Gut Microbes3. (doi:10.4161/gmic. 19896)

36. Chandler JA, Lang J, Bhatnagar S, Eisen JA, Kopp A. 2011 Bacterial communities of diverse Drosophila species: Ecological context of a host-microbe model system. PLoS Genet.7. (doi:10.1371/journal.pgen.1002272)

37. Wong AC-N, Chaston JM, Douglas AE. 2013 The inconstant gut microbiota of Drosophila species revealed by 16S rRNA gene analysis. ISME J. 7, 1922-1932. (doi:10.1038/ismej.2013.86)

38. Chandler JA, James PM, Jospin G, Lang JM. 2014 The bacterial communities of Drosophila suzukii collected fromundamaged cherries. Peer/ 2014, 1-10. (doi:10.7717/peerj.474)

39. Martinez-Sañudo I, Simonato M, Squartini A, Mori N, Marri L, Mazzon L. 2018 Metagenomic analysis reveals changes of the Drosophila suzukirmicrobiota in the newly colonized regions. Insect Sci. 25, 833-846. (doi:10.1111/1744-7917.12458)

40. Crotti E et al. 2010 Acetic acid bacteria, newly emerging symbionts of insects. Appl. Environ. Microbiol.76, 6963-6970. (doi:10.1128/AEM.01336-10)

41. Shin SC, Kim S-H, You H, Kim B, Kim AC, Lee K-A, Yoon J-H, Ryu J-H, Lee W-J. 2011 Drosophila microbiome modulates host developmental and metabolic homeostasis via insulin signaling. Science334, 670-674. (doi:10.1126/science.1212782)

42. Staubach F, Baines JF, Künzel S, Bik EM, Petrov DA. 2013 Host species and environmental effects on bacterial communities associated with Drosophila in the laboratory and in the natural environment. PLoS One8, e70749. (doi:10.1371/journal.pone.0070749)

43. Stefanini I. 2018 Yeast-insect associations: It takes guts. Yeast35, 315-330. (doi:10.1002/yea.3309)

44. Bellutti N, Gallmetzer A, Innerebner G, Schmidt S, Zelger R, Koschier EH. 2018 Dietary yeast affects preference and performance in Drosophila suzukii. J. Pest Sci. (2004). 91, 651-660. (doi:10.1007/s10340-017-0932-2)

45. Mazzetto F, Gonella E, Crotti E, Vacchini V, Syrpas M, Pontini M, Mangelinckx S, Daffonchio D, Alma A. 2016 Olfactory attraction of Drosophila suzukiiby symbiotic acetic acid bacteria. J. Pest Sci. (2004). 89, 783-792. (doi:10.1007/s10340-016-0754-7)

46. Wallingford AK, Hesler SP, Cha DH, Loeb GM. 2016 Behavioral response of spotted-wing Drosophila, Drosophila suzukii Matsumura, to aversive odors and a potential oviposition deterrent in the field. Pest Manag. Sci.72, 701-706. (doi:10.1002/ps.4040)

47. Knaden M, Strutz A, Ahsan J, Sachse S, Hansson BS. 2012 Spatial representation of odorant valence in an insect brain. Cell Rep. 1, 392-399. (doi:10.1016/j.celrep.2012.03.002)

48. Stensmyr MC et al. 2012 A conserved dedicated olfactory circuit for detecting harmful microbes in Drosophila. Cell 151, 1345-1357. (doi:10.1016/j.cell.2012.09.046)

49. Liu W, Zhang K, Li Y, Su W, Hu K, Jin S. 2017 Enterococci mediate the oviposition preference of Drosophila melanogasterthrough sucrose catabolism. Sci. Rep. 7, 1-14. (doi:10.1038/s41598-017-13705-5)

50. Yang CH, Belawat P, Hafen E, Jan LY, Jan YN. 2008 Drosophila egg-laying site selection as a system to study simple decision-making processes. Science. 319, 1679-1683. (doi:10.1126/science.1151842)

51. Joseph RM, Devineni A V., King IFG, Heberlein U. 2009 Oviposition preference for and positional avoidance of acetic acid provide a model for competing behavioral drives in Drosophila. Proc. Natl. Acad. Sci. U. S. A. 106, 11352-11357. (doi:10.1073/pnas.0901419106) 


\section{Figure captions}

Figure 1. Experimental scheme of the oviposition assay to quantify response to water-soluble substances deposited by flies on the surface of media. (a) Water-soluble substances are collected from inoculated and control plates. (b) Oviposition assay using media inoculated with solutions from (a) for $24 \mathrm{~h}$.

Figure 2. Comparisons of the preference indices (PIs) of D. melanogaster, D. biarmipes, and D. suzukii for oviposition substrates treated with inoculant from conspecific (open boxplots) or heterospecific (filled boxplots in gray) flies. (a) The PIs assayed on soft substrate $(1 \%$ agar medium) with and without inoculant treatment (microbial growth). (b) The PIs assayed on 1\% agar medium for substrates treated with sterile filtered solutions of inoculant. (c) The PIs assayed on hard oviposition substrate (3\% agar medium) with and without inoculant treatment (microbial growth). Control substrates were treated with solutions from non-exposed (non-inoculated) substrate in all assays. Species used for heterospecific inoculations were conducted using D. suzukii for D. melanogaster assay, and D. melanogaster for D. biarmipes and D. suzukii assays. Results from assays with fewer than 10 eggs on either substrate were excluded from the analysis. Box signifies the upper and lower quartiles and horizontal bar indicates median. Upper and lower whiskers represent maximum and minimum $1.5 \times$ interquartile range, respectively. The difference from PI $=0$ (no preferences) was tested by Wilcoxon signed rank test with Bonferroni correction for multiple comparisons (6 tests). *: $\mathrm{p}<0.05$, ns: $\mathrm{p} \geq 0.05$.

Figure 3. Preference indices (PIs) for the soft substrate with and without microbes. (a) The substrate placement in the chambers for the oviposition assay. " $1 \%$ " and " $3 \%$ " indicate soft $(1 \%$ agar medium) and hard (3\% agar medium) oviposition substrates, respectively. The microbe (+) chambers have been treated with inoculant collected from substrate surface exposed to $D$. melanogaster; microbial (-) chambers were treated with inoculant from non-exposed surfaces. (b) The preference indices (PI) for soft oviposition substrate in the absence (open boxplots) and presence (filled boxplots in gray) of microbes. Results from assays with fewer than 10 eggs on either substrate were excluded from the analysis. Box signifies the upper and lower quartiles and horizontal bar indicates median. Upper and lower whiskers represent maximum and minimum $1.5 \times$ interquartile range, respectively. Statistical significance was tested by permutation test with Bonferroni correction for multiple comparisons (6 tests). *: p< $0.05, n s: p \geq$ 0.05 . 
(a)

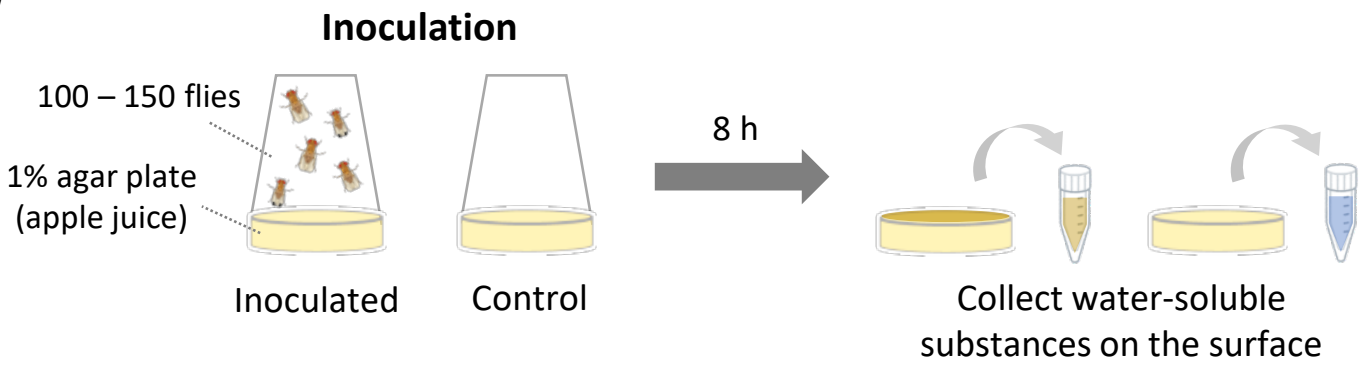

(b)

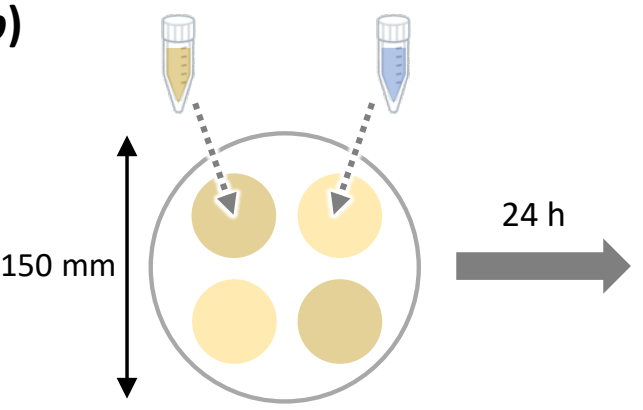

Spread collected aqueous solution onto new media

\section{Oviposition assay}

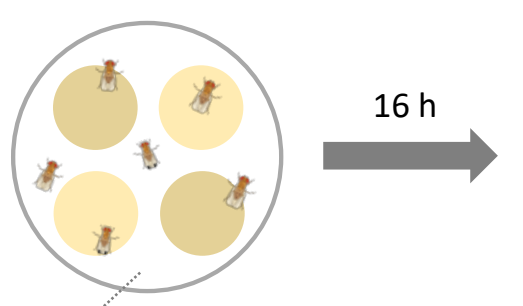

Place 20 females and 10 males
Count the number of eggs on each plate

Figure 1 

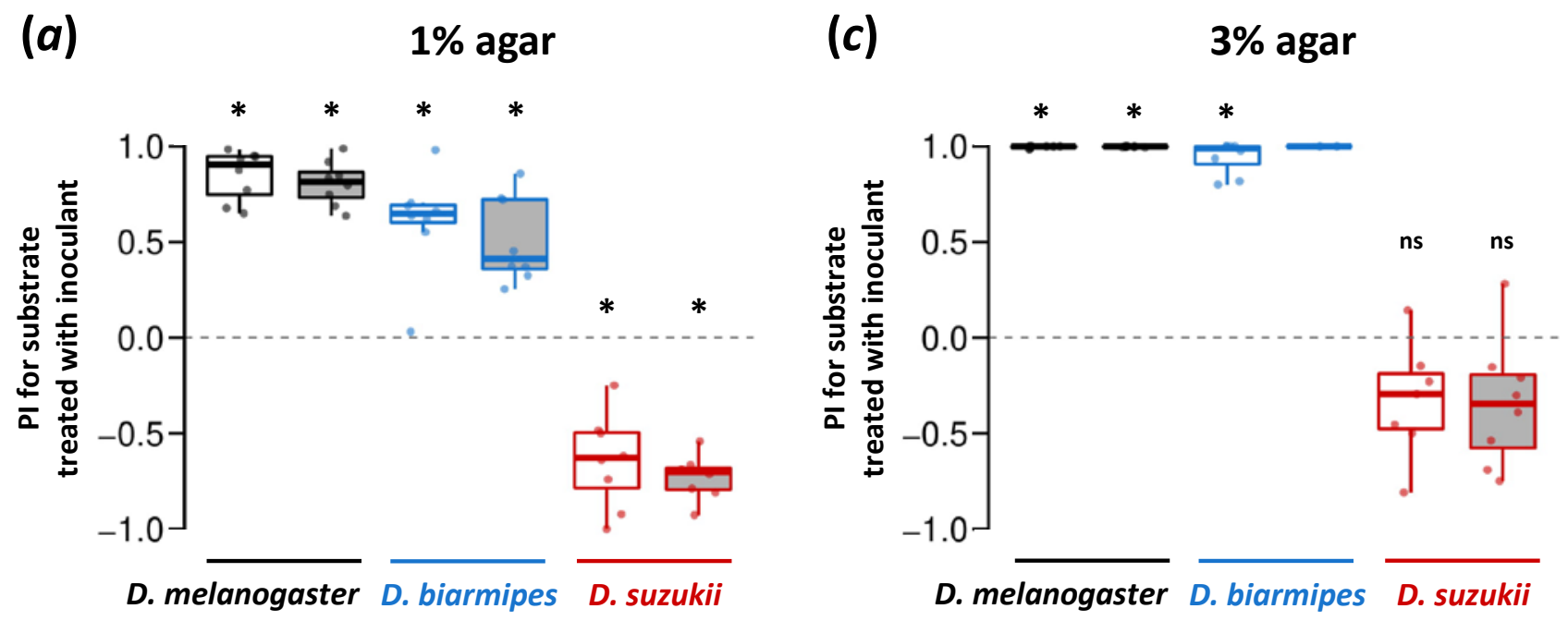

(b)

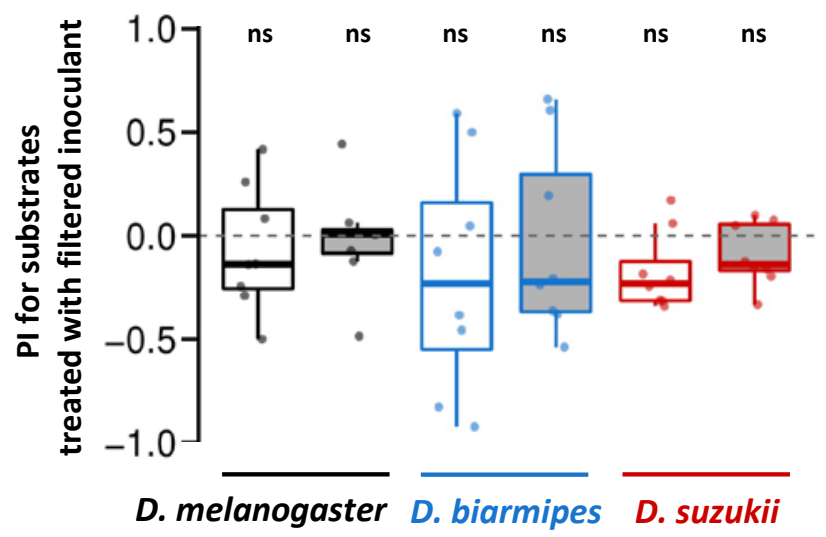

Inoculant source:

Substrate exposed to conspecific individuals

Substrate exposed to heterospecific individuals

Figure 2 
(a) Microbes (-) Microbes (+)
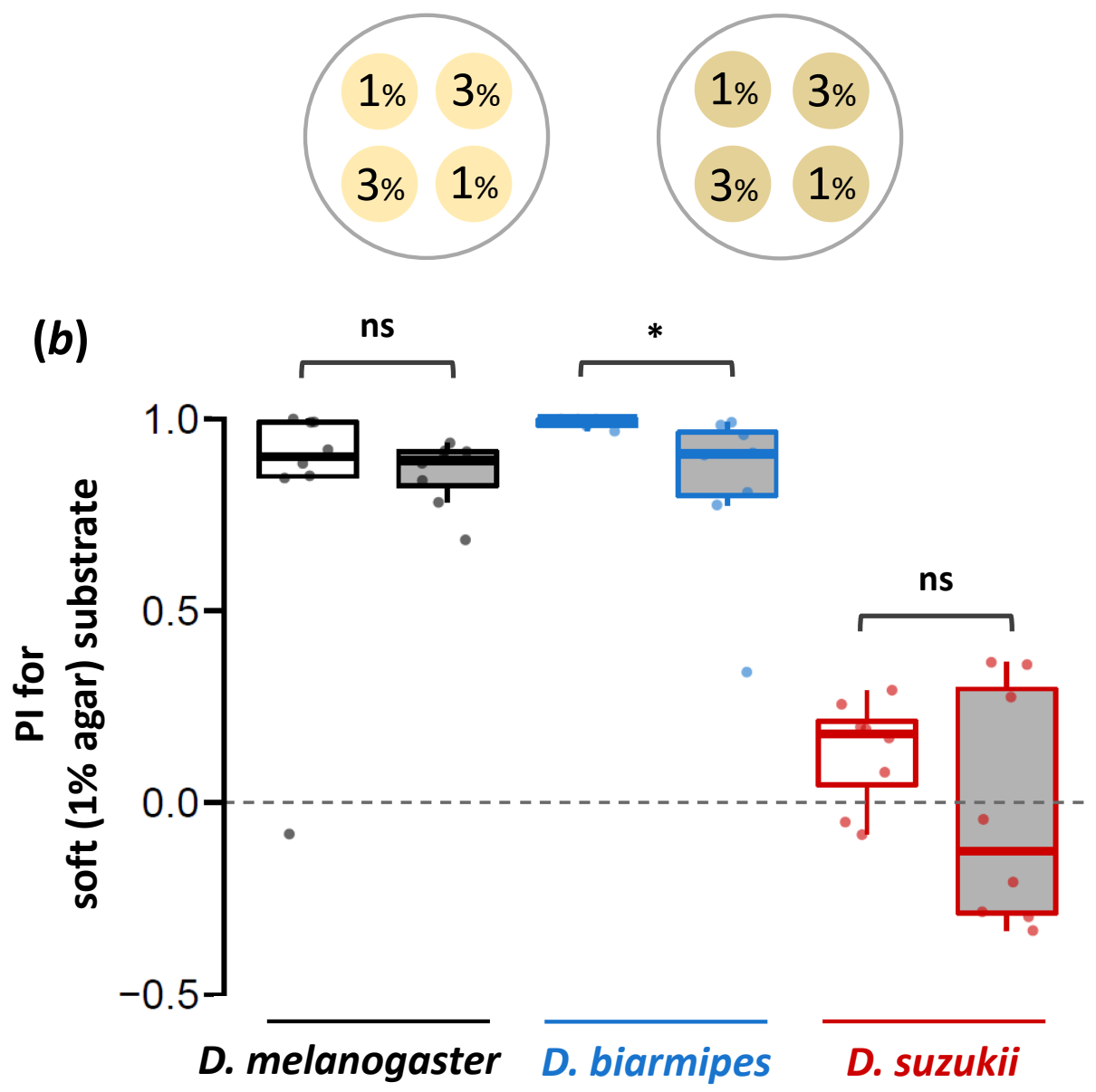

Figure 3 